Journal of the Magnetics Society of Japan Vol. 15 Supplement, No. S2 (1991)

(C) 1991 by The Magnetics Society of Japan

\title{
EFFECT OF OXYGEN GAS ON COLUMNAR STRUCTURES AND MAGNETIC PROPERTIES OF CO-O EVAPORATED THIN FILM
}

Makoto NAGAO, Tadashi YASUNAGA, Hiroaki DOHSHITA, Minoru KANAZAWA and Toshihiko MIURA Reseach and Development Center Magnetic Products Division Fuji Photo Film Co., Ltd. 12-1, Ohgi-cho 2 Chome Odawara, Kanagawa, Japan

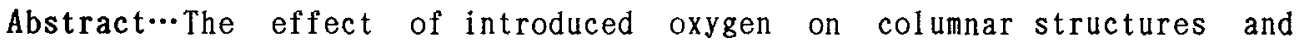
magnetic properties of $\mathrm{Co}-0$ evaporated thin $\mathrm{film}$ prepared with a roll coater has been investigated. When oxygen was introduced close to the end side of the deposition of Co vapour flow, obliquity of columns as well as magnetic anisotropy was observed. On the other hand when an adequate amount of oxygen was introduced close to the beginning side of the deposition of Co vapour flow, the columns and the magnetic anisotropy were almost vertical to the plane of the film. The vertical columnar structure was also observed in the $\mathrm{fi} 1 \mathrm{~m}$ that was prepared using Ar gas, instead of oxygen, introduced close to the beginning side of the deposition. The existence of gas in forming the initial layer is considered to play a clucial role on the thin film growth.
\end{abstract}

\section{INTRODUCTION}

Various types of medium for perpendicular magnetic recording have been investigated $(1,2,3)$. The desirable direction of magnetic anisotropy of the medium depends on types of head. For example, when a single pole head is applied on a doublemagnetic-layer, a perpendicular recording layer and a high permeability layer, the recording layer has to exhibit pure perpendicular magnetic anisotropy for high recording density(1), but when a ring head is applied on a single magnetic layer, a medium with small obliquity magnetic anisotropy is reportedly advantageous to attain single-peak-1ike wave form with narrow pulse width and a high output (4, 5). The optimum direction of the magnetic anisotropy have been controlled by the incident angle of Co or Co-Cr vapour flow(4, $5)$.

The present work has aimed to elucidate how the direction of magnetic anisotropy and that of columnar structure of Co-0 thin film depends on the way oxygen gas is applied.

\section{EXPER I MENTAL PROCEDURE}

Co-0 thin films were prepared by reactive evaporation with a roll coater as shown in Fig. 1. Co was evaporated from a crucible by means of electron beam heating in the presence of oxygen gas to be deposited on a $10 \mu \mathrm{m}$ thick PET substrate running on a cooling can. The initial pressure was about $10^{-5}$ torr. $0 x y g e n$ partial 
pressure was from $10^{-4}$ to $10^{-5}$ torr. The deposition rate of Co was around $200 \mathrm{~nm} / \mathrm{sec}$. Two masks were provided to restrict the incident angle of Co vapour flow within the range of $40^{\circ}$ to $20^{\circ}$. The composition of the Co-0 film was controlled by the flow of oxygen gas. The flow rate of oxygen gas was from $200 \mathrm{cc} / \mathrm{min}$. to $800 \mathrm{cc} / \mathrm{min}$. The oxygen gas was introduced in vicinities of the two masks, one at end side of deposition ( $A$ in Fig. 1) and the other at beginning side of deposition(B in Fig. 1). The thickness of Co$0 \mathrm{film}$ was from $120 \mathrm{~nm}$ to $230 \mathrm{~nm}$. Magnetic properties of the Co-0 films and depth profiles of oxygen and cobalt of the films were measured by vibrating sample magnetometery (VSM) and Auger electron spectroscopy (AES) respectively. Columnar structure of the films was studied using transmission electron microscopy (TEM).

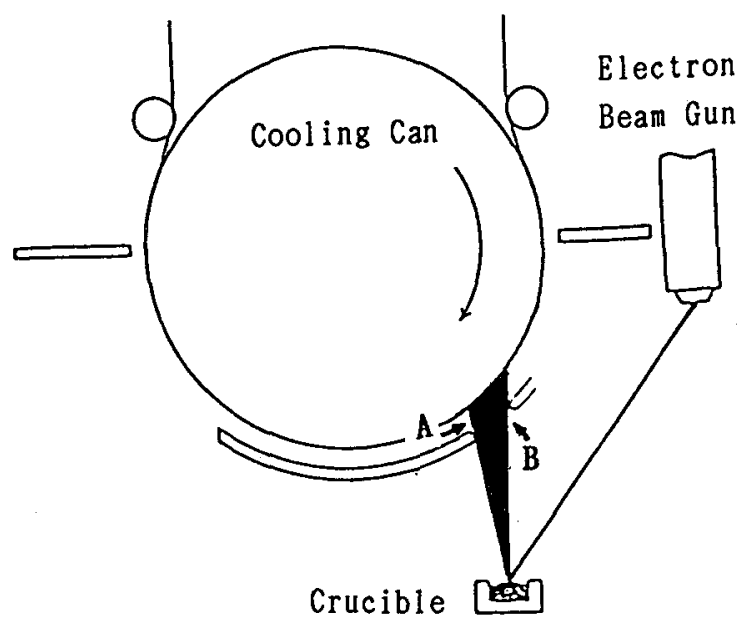

Fig..1 Schematic diagram of experimental apparatus

\section{RESULTS AND DISCUSSION}

Case 1:0xygen introduced from $A$ in Fig. 1.

The depth profiles of oxygen and cobalt and TEM cross-section views along to longitudinal direction in the co-0 film are shown in Fig.2. Most of oxygen exists near the surface of the film, whereas there is also a considerable amount of oxygen near the PET substrate.

a)oxygen content :15 at\%
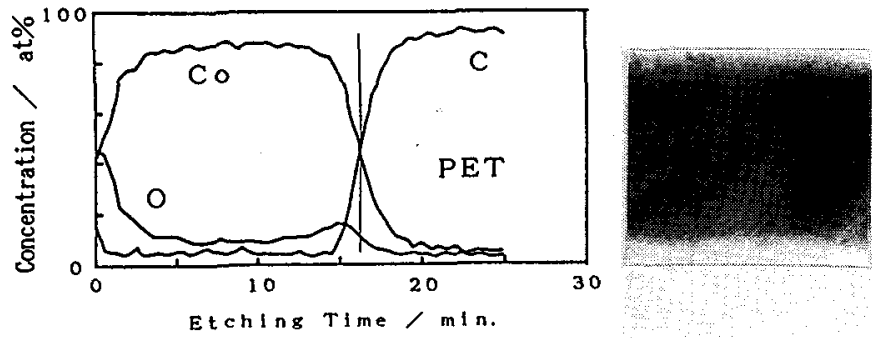

b) oxygen content :20 at\%
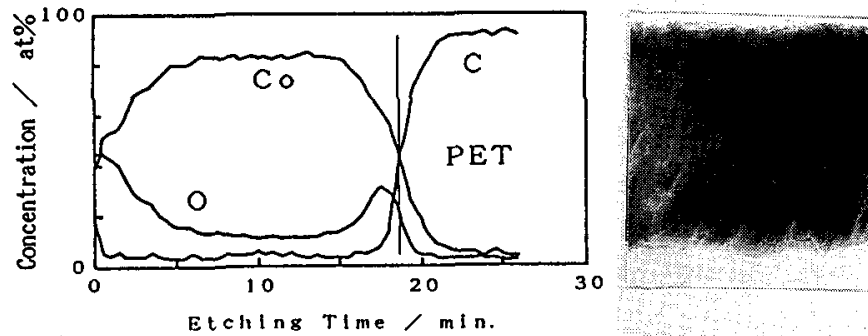

c) oxygen content :26 at $\%$
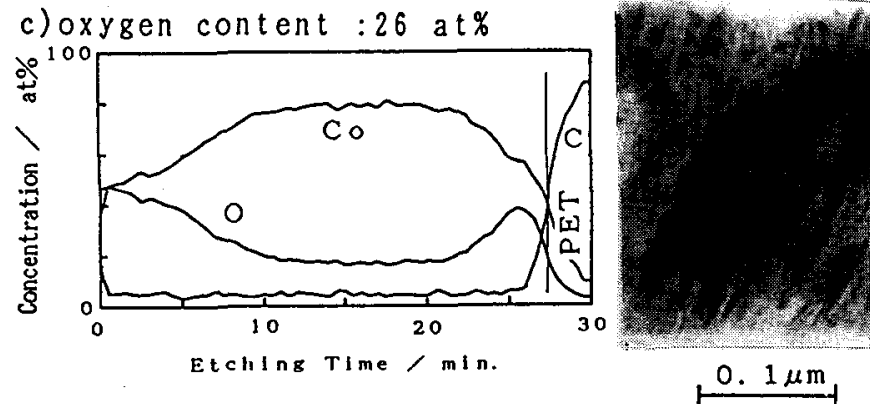

FIG. 2 Depth profile and TEM cross-section view along to the longitudinal direction in the Co-0 thin film

The co-0 thin film is composed of fine grains that are seemed to form a columnar structure. The observed oblique angle between the column and the normal of the film plane vs. oxygen content is shown in Fig. 3. The anglesare constant at about $30^{\circ}$ irrespective of the oxygen content. 


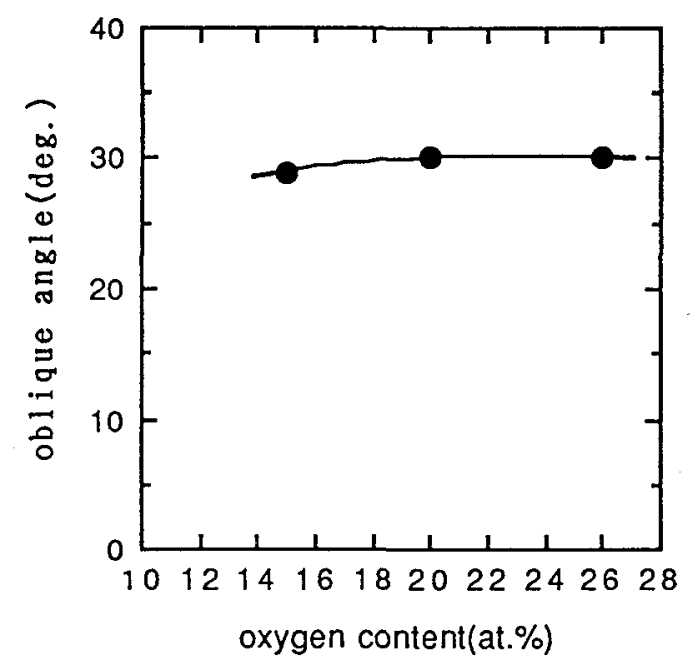

Fig. 3 Oblique angle between the column and the normal of the film plane vs. oxygen content

The ratio of residual magnetization along longitudinal to transverse directon $\mathrm{Mr}(\mathrm{LD}) / \mathrm{Mr}$ (TD) and that of coecivity Hc(LD) /Hc(TD) vs. oxygen content are shown in Fig. 4. They increase with the oxygen content. This means that the magnetic anisotropy along the longitudinal direction is enhanced by the oxygen content.

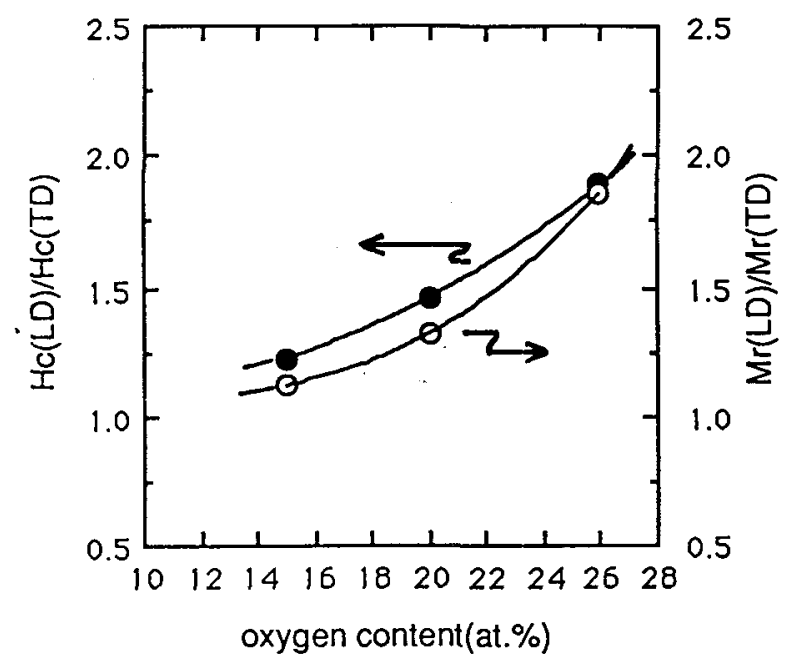

Fig. $4 \mathrm{Mr}(\mathrm{LD}) / \mathrm{Mr}$ (TD) and $\mathrm{Hc}(\mathrm{LD}) / \mathrm{Hc}$ (TD) vs. oxygen content
Angular dependence of coercivity of the Co-0 films, with oxygen content as a parameter, is shown in Fig. 5. The abscissa. $\theta$, denotes the angle of applied magnetic field direction to the normal of the film plane. Coercivity increases with the oxygen content, whereas patterns of angular dependence of $\mathrm{Hc}$ are similar to that of obliquely evaporated MEtape(6). This result suggests that the direction of magetic anisotropy does not changed but the strength of the magnetic anisotropy is enhanced by increasing oxygen content.

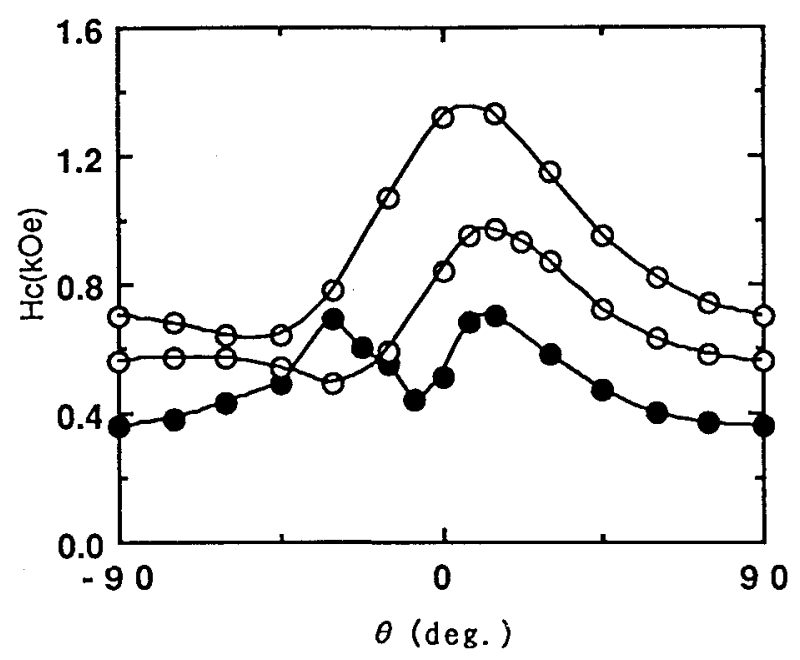

Fig. 5 Angular dependence of HC

Case 2 :0xygen introduced from $B$ in Fig. 1. The depth profiles of oxygen and cobalt and TEM cross-section views along to the longitudinal direction in the Co-O film are shown in Fig. 6. In all films, majority of oxygen exists near the PET substrate.

The obliquity of the column vs. oxygen content is shown in Fig. 7. When the oxygen content is small, for example 12 at. $\%$, columns are oblique to the plane by $20^{\circ}$. As the oxygen content increases, the angle becomes smaller. 
a) oxygen content :12 at\%

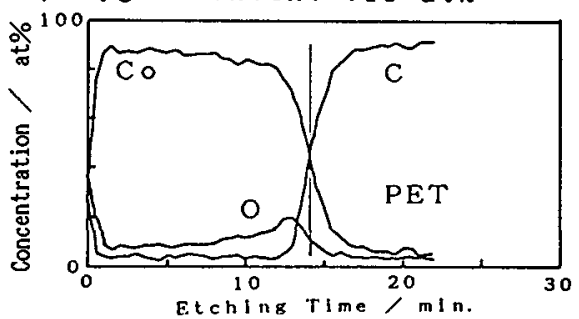

b)oxygen content :14 at

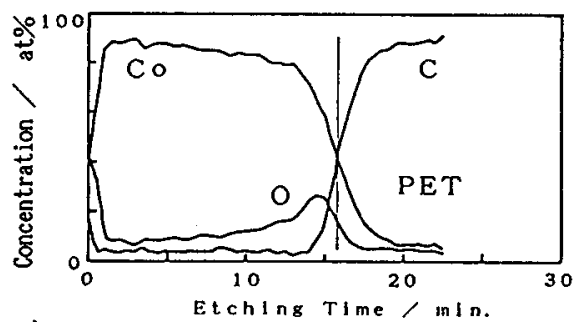

c) oxygen content : 22 at $\%$

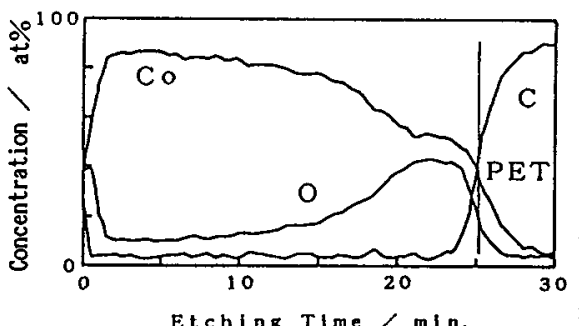

FIG. 6 Depth profile and TEM cross-section view along to the longitudinal direction in the Co-O thin $\mathrm{fi} / \mathrm{m}$

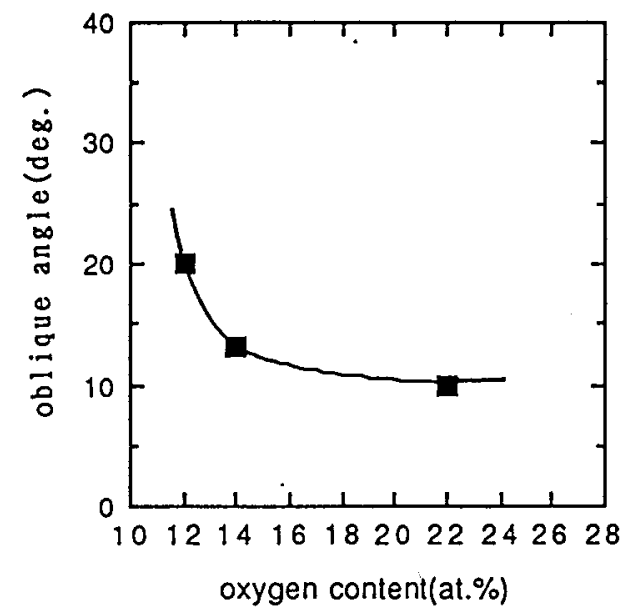

Fig. 7 oblique angle between the column and the normal of the film plane

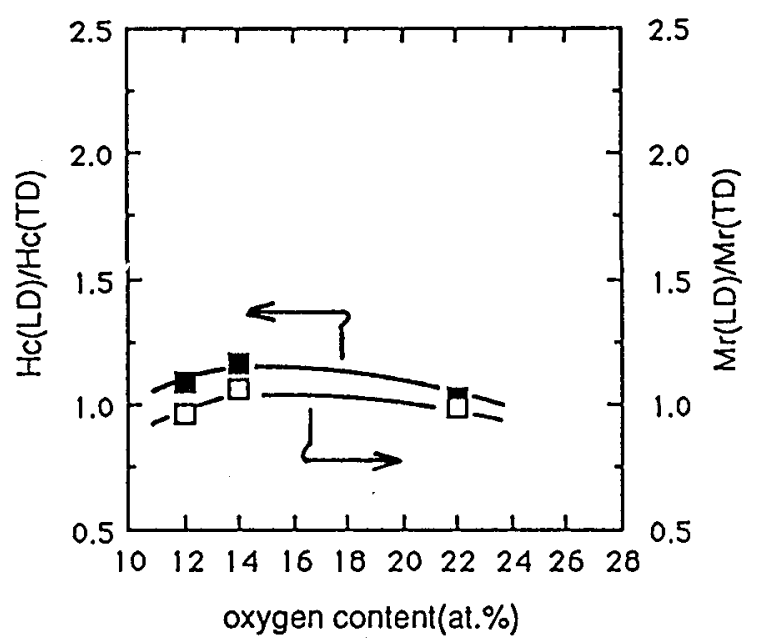

Fig.8 $\mathrm{Mr}(\mathrm{LD}) / \mathrm{Mr}(\mathrm{TD})$ and $\mathrm{Hc}(\mathrm{LD}) / \mathrm{Hc}(\mathrm{TD})$ vs. oxygen content

$\mathrm{Mr}(\mathrm{LD}) / \mathrm{Mr}$ (TD) and $\mathrm{HC}(\mathrm{LD}) / \mathrm{Hc}$ (TD) vs. oxygen content are shown in Fig. 8. Both of them are around at about 1.0 independent of the oxygen content. This means that the magnetic properties within the film plane are almost isotropic.

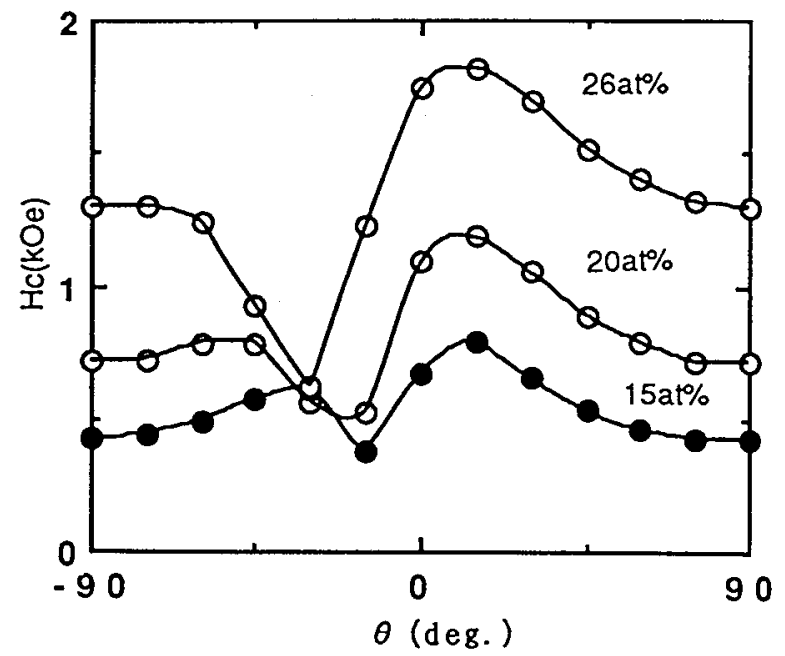

Fig. 9 Angular dependence of $\mathrm{HC}$

Angular dependence of $\mathrm{Hc}$ on oxygen content are shown in Fig. 9. The sample with oxygen content, 12 at.\%, exhibits low Hc and double peaks. As the oxygen content increases, Hc becomes higher and the patterns of angular dependence of Hc change. 
When the oxygen content reaches 22 at. $\%$. Hc is as high as 1350 oe and the pattern looks like a single-peak wave.

This result indicates that the magnetic anisotropy is enhanced and each direction approaches to the normal of the film plane with increasing the oxygen content.

Case 3 : Ar introduced from B in Fig. 1.

In order to investigate the reason why the direction of the columns and the magnetic anisotropy are almost vertical to the film plane in spite of oblique evaporation, Ar, instead of oxygen, was introduced from $B$ in Fig. 1. The partial Ar pressure was about $10^{-3}$ torr. The flow rate of Ar gas was $280 \mathrm{cc} / \mathrm{min}$.

The TEM cross-section view of the film is shown in Fig. 10. The weaker columnar structure can be observed, compared with Co $-0 \mathrm{film}$. They stand almost vertical to the film plane, while the magnetic properties are similar to Co-0 with small oxygen content. It shows that Ar gas has important effect on the columnar structure but has smaller effect on the magnetic properties, compared with the case of oxygen.

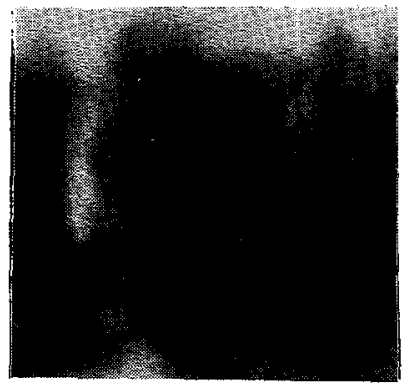

$0.1 \mu \mathrm{m}$

FIG.2 Depth profile and TEM cross-section view along to the longitudinal direction in the Co-O thin film
The reason why the columnar structures and the magnetic properties depend on the way oxygen was introduced has not been clarified yet. 0 ur result indicates that the content of oxygen is not a single limiting factor. The direction of Co-Cr vapour flow in making initial layer is reportedly a dominant factor to a thin film growth(7), while our results show that the existence of gas just in forming initial layer irrespective of reactive gas or not play a crucial role on the direction of column. The existence of gas may disturb self-shadowing growth that has been considered as the origin of the oblique column(8). We suppose that magnetic separation of the columns and that of the grains by oxidation produced the above-mentioned magnetic properties. Further investigation, especially on the role of the direction of introduced gas, should be needed to clarify the mechanism.

\section{CONCLUSIONS}

- The columnar structure and magnetic properties of Co-0 evaporated thin film depends on the way oxygen is applied.

- Thus when oxygen was introduced close to the end side of deposition of Co vapour flow, obliquity of columns as well as magnetic anisotropy were observed.

- When an adequate amount of oxygen gas was introduced close to the beginning side of the deposition of Co vapour flow, the columns and the magnetic anisotropy were almost vertical to the film plane.

- The vertical columnar structure was also observed in the film that was prepared using Ar gas, instead of oxygen, introduced close to the beginning side of the deposition. 
- The existence of gas in forming initial layer play a crucial role on the thin film growth.

\section{ACKNOWLEDGEMENTS}

The authors would like to thank K. Sano of Fuji Photo Film Co., Ltd. for his helpful discussions and encouragement.

\section{REFERENCES}

[1]Y. Nakamura, I. Tagami, IEEE Trns. Mag., $M A G-24,2329(1988)$

[2] J. Hokkyo, J. Kugiya, J. Mag. Soc. Jpn. Vol. 13 , Supplement, S1, 1(1989)

[3]Y. Ouchi, J.Mag. Soc. Jpn. Vol.13, Supplement, $S 1,611(1989)$

[4]R. Sugita, N. Echigo, K. Tohma, C. Yamamitsu, IEEE Trans. Mag., MAG-26, 286, (1990)

[5]Y. Tateno, K. I wasaki, H. Naruse, R. Chubachi, IEEE. Trans. Mag. , MAG-25,4186. (1989)

[6] K. Shinohara, H. Yoshida, M. Odagi ri, A. Tomago IEEE.Trans.Mag., Vo1.MAG-20,824. (1984)

[7]R. Sugita, IEEE. Trans. Mag., MAG-20,687, (1984)

[8]D. 0. Smith, M. S. Cohen, G. P. Weiss, J.Appl. Phys., 31.1755(1960) 